\title{
Personalidad y agotamiento emocional académico en estudiantes universitarios peruanos: un estudio predictivo
}

\author{
Rony Prada-Chapoñan \\ Unidad de Investigación de la Facultad de Humanidades, Universidad Señor de Sipán, Chiclayo, Perú \\ https://orcid.org/0000-0002-4268-6325
}

Jhonatan S. Navarro-Loli

Instituto de Investigación de Psicología, Universidad de San Martín de Porres, Lima, Perú https://orcid.org/0000-0001-6264-3157

Sergio Dominguez-Lara*

Instituto de Investigación de Psicología, Universidad de San Martín de Porres, Lima, Perú Vicerrectorado de Investigación, Universidad Privada San Juan Bautista, Lima, Perú https://orcid.org/0000-0002-2083-4278

Recibido:20/08/20 Revisado: 10/09/20 Aceptado:24/09/20 Publicado:3/11/2020

\section{Resumen}

La personalidad es una característica relevante para predecir la conducta académica del estudiante, y su asociación con el agotamiento emocional académico aún es un tópico reciente. El propósito de la presente investigación fue analizar la capacidad predictiva de los cinco grandes factores de personalidad sobre el agotamiento emocional académico en universitarios, en una muestra de 453 estudiantes (60.9\% mujeres) entre 17 y 40 años de edad, quienes fueron evaluados con la Escala de Cansancio Emocional y el Big Five Inventory-15p. Fue implementado un análisis de regresión múltiple para agotamiento emocional académico con los rasgos de personalidad como predictores. Los resultados indican que los rasgos de personalidad explican significativamente el agotamiento emocional $\left(R^{2}>20\right)$, y el neuroticismo, extraversión y responsabilidad predicen el agotamiento emocional académico. En conclusión, la capacidad predictiva de los rasgos de personalidad sobre el agotamiento emocional académico es significativa. Se discuten las consecuencias prácticas y las limitaciones del estudio.

Palabras clave: Personalidad; Agotamiento emocional académico; Estudiantes universitarios

\section{Personality y academic emotional exhaustion in peruvian college students: a predictive study}

\footnotetext{
Abstract

Personality is a relevant feature to predict the student's academic behavior, and its association with academic emotional exhaustion is still a recent topic. The aim was determine the predictive ability of personality on academic emotional exhaustion in 453 college students (60.9\% women) between ages 17 and 40 years old, who were assessed with the Emotional Exhaustion Scale and the Big Five Inventory-15p. Was implemented a regression multiple analysis on emotional exhaustion,
} 
with the personality traits as predictors. The findings shows that the personality traits explain significantly the emotional exhaustion $\left(R^{2}>.20\right)$, and the neuroticism, extraversion and consciousness predict the emotional exhaustion. In conclusion, the predictive ability of personality on academic emotional exhaustion is significant. The practical consequences and limitations are discussed.

Keywords: Personality; Academic emotional exhaustion; College students

\title{
Personalidade e exaustão emocional acadêmica em universitários peruanos: um estudo preditivo
}

\begin{abstract}
Resumo
A personalidade é uma característica relevante para prever o comportamento acadêmico do aluno, e sua associação com a exaustão emocional acadêmica ainda é um tópico recente. 0 objetivo da presente investigação foi analisar a capacidade preditiva dos cinco fatores de personalidade na exaustão emocional acadêmica de universitários em uma amostra de 453 estudantes (60,9\% mulheres) entre 17 e 40 anos, avaliados com a Escala de Exaustão Emocional e o Big Five Inventory-15p. Uma análise de regressão múltipla para exaustão emocional acadêmica foi implementada, com traços de personalidade como preditores. Os resultados indicam que os traços de personalidade explicam significativamente a exaustão emocional $\left(R^{2}>.20\right)$, e o neuroticismo, a extroversão e a responsabilidade predizem a exaustão emocional acadêmica. Concluindo, a capacidade preditiva dos traços de personalidade no esgotamento emocional acadêmico é significativa. As conseqüências práticas e limitações do estudo são discutidas.
\end{abstract}

Palavras-chave: Personalidade; Exaustão emocional acadêmica; Estudantes universitários

Citar como:

Prada-Chapoñan, R., Navarro-Loli, J., \& Dominguez-Lara, S. (2020). Personalidad y agotamiento emocional académico en estudiantes universitarios peruanos: un estudio predictivo. Revista Digital de Investigación en Docencia Universitaria, 14(2), e1227. https://doi.org/10.19083/ridu.2020.1227

\section{Introducción}

Recientemente, el estudio del burnout académico (BA) ha cobrado mayor importancia (Martínez y Marques, 2005) debido a las consecuencias personales y académicas que trae consigo (Escuderos, Colorado, y Sañudo, 2017). Su estudio fue posible porque Schaufeli, Martínez, Marques, Salanova y Bakker (2002) reportaron que las dimensiones del burnout, normalmente observadas en el ámbito laboral, también están presentes en el contexto universitario, ya que los estudiantes pueden desarrollar burnout a causa de sus estudios. Entonces, considerando lo anterior y que en la universidad el alumno está expuesto a factores similares a los del ámbito laboral (espacio físico al que asiste diariamente, exigencias, trabajos, objetivos que cumplir, etc.), es posible desencadenar burnout (Salanova, Bresó, \& Schaufeli, 2005; Salanova Salanova, Schaufeli, Martínez, \& Bresó, 2010).

De este modo, el agotamiento emocional (AE) es producto de las exigencias académicas, que deriva posteriormente en una actitud cínica y de escaso interés por los estudios y finalmente en creencias de baja eficacia personal. Este hallazgo ha sido sustentado al identificar que los obstáculos personales, sociales y organizacionales en el contexto universitario son predictores positivos del BA, mientras que los facilitadores personales, sociales y organizacionales predicen el BA negativamente (Salanova et al., 2010), dado que las 
experiencias de fracaso o de ausencia de destreza en algunos aspectos conllevan al BA, concebido como una crisis de eficacia (Salanova, Martínez, Bresó, Llorens, \& Grau, 2005).

El planteamiento actual de Maslach y Leiter (2016), aunque enfocado en entornos laborales, argumenta que el AE es la primera dimensión del burnout en desarrollarse como respuesta a las exigencias y la sobrecarga por ser una respuesta básica al estrés (Maslach, 2003), y que posteriormente llevaría a consolidar el burnout. Asimismo, en el ámbito académico el AE aumenta a medida que avanza el ciclo académico (Galbraith \& Merri1l, 2012), lo que presenta una asociación implícita con la dificultad de las asignaturas y el hecho de afrontar evaluaciones periódicas. Por tales motivos se considera al AE como el núcleo (core) del burnout (Salanova et al., 2005), postulado que concuerda con aproximaciones teóricas como las de Kristensen, Borritz, Viladse y Christensen (2005). Por ello, la presente investigación considera al AE como un constructo unidimensional, representativo del BA y que puede ser estudiado en el contexto educativo (Nikodijević, Labrović, \& Đoković, 2012; Tuithof et al., 2017).

En los estudios del burnout académico, la investigación del agotamiento emocional se ha centrado en la identificación de variables asociadas a su desarrollo (variables del contexto académico, del ámbito social e interpersonales) y sus consecuencias, tales como el bajo rendimiento académico, alteraciones en la salud física y mental, así como el consumo excesivo de alcohol, ansiedad y depresión, etc. (Caballero, Hederich, \& Palacio, 2010; Galbraith \& Merrill, 2015; Jackson et al., 2016; Pagnin et al., 2014; Pereira-Lima \& Loureiro, 2015). Tomando en cuenta las variables que propician el desarrollo del burnout académico, existen propuestas que sugieren que el punto de partida es la falta de equilibrio entre las exigencias del contexto académico y los recursos del estudiante (Caballero et al., 2010); además de ser visto como el producto de un afrontamiento deficiente ante eventos percibidos como adversos en la vida académica (Arias-Gundín \& Vizoso, 2018), por lo que cobrarían relevancia las variables personales en el estudio del agotamiento emocional.

Desde el factor personal, se han identificado algunos correlatos relevantes vinculados con la esfera emocional del individuo. Por ejemplo, una regulación emocional deficiente se asocia con mayores síntomas de agotamiento emocional (González, Souto, Fernández, y Freire, 2011; Duru, Duru, \& Balkis, 2014). Así también, el uso frecuente de estrategias de regulación emocional tales como autoculparse, rumiar y catastrofizar, intensifican el agotamiento emocional y propician el desarrollo de burnout académico (Dominguez-Lara, 2018). Además, el uso de estrategias de afrontamiento como la evitación emocional (Palacios, Caballero, González, Gravin, \& Contreras, 2012), las creencias de ineficacia académica (Palacios et al., 2012; Salanova et al., 2005; Yu, Chae, y Chang, 2016), el locus de control externo y la baja autoestima (Ramos, Manga, \& Morán, 2005), son fuertes predictores del agotamiento emocional y estrés académico. Asimismo, se sugiere que el desarrollo del burnout se encuentra ligado a características de personalidad (Alarcon, Eschleman, y Bowling, 2009; David, 2010; García-Izquierdo, Ramos-Villagrasa, y García-Izquierdo, 2009; Polo, Santiago, Navarro, y Alí, 2016), por lo que es necesario estudiar su relación en el ámbito académico (Ferrel, Ferrel, Cantillo, Jaramillo, \& Jiménez, 2017).

La influencia de la personalidad sobre el burnout académico puede explicarse en el marco del sistema cognitivo-afectivo de la personalidad (Mischel \& Shoda, 1995), según el cual la personalidad del individuo afecta la forma en la que se evalúa la información procedente del entorno. De este modo, las personas son capaces de codificar sus creencias, reacciones, y planes de autorregulación para controlar sus conductas impulsivas, frustraciones y miedos. Así, estas codificaciones se denominan unidades cognitivo-afectivas que sirven para explicar las relaciones entre personalidad y conducta (Mischel \& Ayduk, 2002). Entonces, aplicándolo al contexto universitario, según la estructura de personalidad del estudiante, este interpretaría las señales del entorno académico y reaccionaría según dicha evaluación. Por ejemplo, una persona con un grado elevado de neuroticismo interpretaría un número determinado de tareas o exámenes como algo en extremo amenazante y desestabilizarse por ese motivo, mientras que alguien con un menor grado de neuroticismo sería capaz de establecer un plan de acción para afrontar de forma exitosa esa exigencia (Armon, 
Shirom, \& Melamed, 2012).

Entonces, en este panorama, se considera al modelo de los cinco grandes factores (5GF; Benet-Martínez \& John, 1998) como un marco de referencia que brinda información descriptiva de diversos rasgos (extraversión, afabilidad, responsabilidad, neuroticismo y apertura) y presenta suficiente evidencia empírica en diversos ámbitos de aplicación de la psicología, incluyendo su asociación con variables vinculadas con el comportamiento académico de estudiantes universitarios. Por ejemplo, se ha documentado relaciones de dichos rasgos con la autoeficacia académica (responsabilidad y apertura; Caprara, Vecchione, Alessandri, Gerbino, y Barbaranelli, 2011; McIIloy, Poole, Ursavas, \& Moriarty, 2015), rendimiento académico (neuroticismo y responsabilidad; De Feyter, Caers, Vigna, \& Berings, 2012), procrastinación académica (responsabilidad y afabilidad; Boysan, y Kiral, 2016), motivación académica (neuroticismo y responsabilidad; Clark y Schroth, 2010; McGeown et al., 2014), entre otras. En este sentido, actualmente se han realizado algunos esfuerzos por conocer la asociación entre personalidad y burnout académico (Caballero et al., 2010), aunque desde otros marcos conceptuales, como el modelo de personalidad biopsicosocial de Cloninger, se sugiere que los individuos que inhiben su comportamiento para evitar el daño y quienes poseen un bajo nivel de autonomia o autodirección tienen mayor predicción al burnout (Lee, Choi, \& Chaeb, 2017); de igual manera, poseer características del tipo de personalidad tipo $\mathrm{D}$ incrementa el riesgo de padecer agotamiento (Skodova, Lajciakova, \& Banovcinova, 2017); sin embargo, no existen estudios en el contexto peruano.

De manera específica se ha logrado identificar que el neuroticismo (García-Izquierdo et al., 2009; Langelaan, Bakker, van Doornen, y Schaufeli, 2006; Morgan y De Bruin, 2010; Sulea, van Beek, Sarbescu, Virgia, y Schaufeli, 2015), el temperamento negativo (Jacobs y Dodd, 2003) y la ansiedad (Cebrià et al., 2001) son predictores del agotamiento emocional, aunque es probable que responsabilidad y apertura puedan predecir negativamente el burnout académico debido a la relación directa de estos rasgos con las creencias sobre la conducta académica del estudiante por ejemplo autoeficacia académica. Por otro lado, se ha encontrado que la personalidad resistente posee un efecto modulador sobre el burnout (da Silva et al., 2014; Garrosa, Rainho, Moreno-Jiménez, y Monteiro, 2010; Moreno, Morett, Rodríguez, y Morante, 2006; Ortega, Ortiz, y Coronel, 2006), es decir, que su presencia puede facilitar o minimizar la presencia de este último; sin embargo, las características de personalidades saludables podrían incluso experimentar burnout académico cuando los estudiantes están expuestos a demandas académicas desafiantes y competitivas por un largo período de tiempo (Lee et al., 2017), incluso si las estrategias empleadas por el docente favorece el involucramiento del estudiante (Hortigüela \& Pérez-Pueyo, 2016).

Por último, con base en estudios previos, se puede afirmar que la personalidad es un factor importante en el desarrollo del agotamiento emocional; no obstante, el estudio de ambas variables de forma conjunta suele pasarse por alto (Morgan \& De Bruin, 2010). De esta manera, el objetivo del presente trabajo fue estudiar la capacidad predictiva de los 5GF sobre el agotamiento emocional en universitarios peruanos, en vista de que la evidencia apunta a que diversos rasgos de personalidad podrían ser factores de riesgo para su desarrollo. Entonces, considerando la evidencia previa, la hipótesis de estudio indica que el rasgo de neuroticismo sería un predictor positivo del agotamiento académico, mientras que la responsabilidad y apertura son predictores negativos.

\section{Método}

\section{Diseño}

Por sus características la presente investigación corresponde a un estudio predictivo transversal (Ato, López, \& Benavente, 2013), en vista de que se analizó la influencia de la personalidad (predictores) sobre el agotamiento emocional académico (criterio).

\section{Participantes}

Fueron evaluados 453 estudiantes (60.9\% mujeres), con edades comprendidas entre 17 y 40 $\left(M_{\text {edad }}=20.89 ; D E_{\text {edad }}=2.688\right)$, de los que el $90.1 \%$ 
fueron menores de 25 años, el 95.4\% era soltero y un $27.4 \%$ reportó que trabaja. Todos ellos cursaban sus estudios en tres carreras profesionales (Administración: 39.5\%; Contabilidad: 42\%; Turismo y Negocios: $17.7 \%$ ) de una universidad de gestión privada ubicada en la zona norte del Perú (región Lambayeque).

\section{Instrumentos}

Big Five Inventory-15p (BFI-15p; Dominguez-Lara y Merino-Soto, 2018a, 2018b). Se trata de una medida breve de los 5GF: Extraversión (e.g., ...es bien hablador), Afabilidad (e.g., ...es generoso), Responsabilidad (e.g., ...persevera hasta terminar el trabajo), Neuroticismo (e.g., ...con frecuencia se pone tenso), y Apertura (e.g., ...valora lo artístico y lo estético) para universitarios peruanos, que tiene 15 ítems con cinco opciones de respuesta (Muy en desacuerdo, Ligeramente en desacuerdo, $\mathrm{Ni}$ de acuerdo ni en desacuerdo, Ligeramente de acuerdo y Muy de acuerdo), a razón de tres ítems por cada dimensión. Los hallazgos de los estudios de validación dan cuenta que se trata de una versión cuyas dimensiones se diferencian apropiadamente unas de otras bajo metodología ESEM, obteniendo a su vez indicadores de fiabilidad relevantes considerando la cantidad de ítems por dimensión (Dominguez-Lara \& Merino-Soto, 2018a, 2018b).

Escala de Cansancio Emocional (ECE, Fontana, 2011). Se utilizó la versión adaptada para estudiantes universitarios peruanos (Dominguez-Lara, Fernández-Arata, Manrique-Millones, Alarcón-Parco, y Díaz-Peñaloza, 2018). Es una escala de autorreporte, que evalúa el agotamiento emocional de forma unidimensional, la cual está compuesta por 10 ítems (e.g., Hay días que noto más la fatiga y me falta energía para concentrarme) en una escala de respuesta de cinco puntos (Raras veces, Pocas veces, Algunas veces, Con frecuencia, y Siempre).

\section{Procedimientos}

Se solicitó el permiso a las autoridades universitarias para evaluar a los estudiantes. Las encuestas fueron administradas en el horario habitual de clase, previa coordinación con los docentes en las semanas iniciales del ciclo académico. Luego de firmar el consentimiento informado, se siguió el procedimiento de aplicación grupal de cuestiona- rios precisando las instrucciones generales y específicas. Así también se enfatizaron los objetivos del estudio, el carácter voluntario de la participación y que esta no sería recompensada. Al término de la evaluación, se agradeció por su colaboración.

En la presente investigación fueron aplicados los principios de la declaración de Helsinki (Asociación Médica Mundial, 1964), así como del código de ética del Colegio de Psicólogos del Perú (2017). Por último, este trabajo es un producto derivado del proyecto Relación entre burnout académico, ansiedad y depresión en estudiantes universitarios: análisis mediacional de factores protectores y de riesgo, aprobado por la universidad del tercer autor.

\section{Análisis de datos}

Inicialmente fue llevado a cabo un análisis descriptivo de los datos, considerando magnitudes en asimetría y de curtosis menores de dos y siete, respectivamente, como un acercamiento razonable a la normalidad univariada (Finney y DiStefano, 2006).

Fue empleado el coeficiente $\alpha$ y la correlación inter-ítem promedio $\left(r_{i i}\right)$ para la confiabilidad de las puntuaciones. En vista de que el BFI-15p evalúa constructos amplios, $\alpha$ con valores alrededor de .60 y $r_{i i}$ en el rango de .15 a .20 son aceptables (Clark \& Watson, 1995).

Posteriormente la muestra fue dividida según sexo a fin de conocer el grado de asociación entre el agotamiento emocional y cada rasgo de personalidad en hombres y mujeres para saber que existe algún tipo de sesgo en el procesamiento, y pueda considerarse más adelante el análisis del grupo total. Desde un enfoque de magnitud del efecto (ES), fueron evaluadas como significativas las correlaciones ( $r$ ) mayores que .20 (Ferguson, 2009). Esto, debido a las limitaciones intrínsecas de la prueba de significancia estadística de la hipótesis nula, sobre todo por su sensibilidad al tamaño muestral derivando en valorar como significativas magnitudes de correlación pequeñas.

Luego de ello, las correlaciones fueron comparadas bajo un enfoque de intervalos de confianza (IC; Zou, 2007; Dominguez-Lara, Moscoso, Merino-Soto, y Navarro-Loli, 2016) y ES mediante el estadístico q (Cohen, 1992), esperando valores > .10 para determinar que existe diferencias entre correlaciones. De este modo, pudo analizarse un 
patrón diferencial de asociación según el sexo.

Para el contraste de la hipótesis de investigación, fue ejecutado un análisis de regresión múltiple con la muestra completa utilizando tres de los cinco rasgos de personalidad (extraversión, responsabilidad y neuroticismo) como predictores y al agotamiento emocional como criterio. En vista de que los coeficientes de confiabilidad fueron de baja magnitud debido, fundamentalmente, a las características del constructo evaluado y la cantidad de ítems (Lang, John, Lüdtke, Schupp, \& Wagner, 2011; Rammstead, \& Beierlein, 2014), el procedimiento de regresión contempló la corrección por atenuación de las correlaciones y el uso de un enfoque bootstrap mediante el uso de una sintaxis en SPSS orientada a ello (Lorenzo-Seva, Ferrando, \& Chico, 2010).

De forma preliminar se analizaron los supuestos estadísticos para ejecutar la regresión lineal. En primer lugar, la linealidad se analizó explorando la asociación entre las variables; la independencia de errores se valoró con la magnitud del estadístico de Durbin-Watson, considerando aceptables los valores entre 1.5 y 2.5 ; la distribución de los residuos se analizó con la prueba de Kolmogorov-Smirnov; y, finalmente, la multicolinealidad se analizó con los indicadores de tole- rancia y el factor de inflación de varianza (FIV), donde valores mayores que .10 y menores que 10, respectivamente, son indicadores de ausencia de multicolinealidad (Vilà Baños, Torrado-Fonseca, \& Reguant-Álvarez, 2019).

Con relación a los resultados, fueron interpretados el coeficiente de determinación $\left(R^{2}\right)$, que refleja la varianza del agotamiento emocional explicada por los $5 \mathrm{GF}$, y si esta fuera significativa, se valora a su vez la magnitud de los coeficientes de regresión estandarizados $(\beta)$ y los coeficientes de estructura $\left(r_{s}\right)$, que son menos afectados por la colinealidad entre predictores y reflejan de forma más precisa la capacidad predictiva de estos sobre la variable criterio (Courville, y Thompson, 2001). Cabe precisar que tanto el $R^{2}$ como el $\beta$ fueron analizados bajo un enfoque de ES.

En cuanto al $R^{2}$, fue considerado pequeño (.02), mediano (.13) y grande (.26) (Ellis, 2010), agregando además su IC; es decir, si el límite inferior del IC de $R^{2}$ es mayor que .02, es relevante. Finalmente, $\operatorname{los} \beta>$.20 fueron significativos (Ferguson, 2009), tomando en cuenta a su vez el IC del mismo modo que con el $\mathrm{R}^{2}$. En general, todos los IC fueron calculados con un enfoque bootstrap (número de replicaciones: 500), valorando el estadístico como significativo si el IC no incluye al cero.

Tabla 1

Análisis descriptivo, distribucional, correlacional y comparativo

\begin{tabular}{|c|c|c|c|c|c|c|c|c|c|c|c|c|}
\hline & \multicolumn{4}{|c|}{$\begin{array}{c}\text { Análisis descriptivo y } \\
\text { normalidad }\end{array}$} & \multicolumn{4}{|c|}{$\begin{array}{l}\text { Correlación entre } \\
\text { predictores }\end{array}$} & \multicolumn{4}{|c|}{ Comparación de correlaciones } \\
\hline & M & $\mathrm{DE}$ & $g_{1}$ & $g_{2}$ & 1 & 2 & 3 & 4 & $\mathbf{r}_{\text {mujeres }}$ & $\mathbf{r}_{\text {varones }}$ & $\mathrm{ICr}_{\mathrm{m}}-\mathrm{r}{ }_{\mathrm{v}}$ & q \\
\hline \multicolumn{13}{|l|}{ Personalidad } \\
\hline 1. Extraversión & 11.409 & 2.238 & -.684 & .434 & 1 & & & & -206 & -.232 & $\begin{array}{r}-.156 \\
.204\end{array}$ & .03 \\
\hline 2. Afabilidad & 12.731 & 2.028 & -1.134 & 1.872 & .461 & 1 & & & -.067 & -.202 & $\begin{array}{r}-.052 \\
.316\end{array}$ & .13 \\
\hline 3. Responsabilidad & 12.067 & 2.169 & -.748 & .746 & .419 & .514 & 1 & & -.182 & -.197 & $\begin{array}{r}-.169 \\
196\end{array}$ & .02 \\
\hline 4. Neuroticismo & 8.171 & 2.816 & .252 & -.412 & -.141 & -.040 & -.003 & 1 & .408 & .328 & $\begin{array}{r}-.083 \\
.247\end{array}$ & .07 \\
\hline 5. Apertura & 11.084 & 2.357 & -.546 & .094 & .316 & .439 & .472 & .039 & -.157 & .061 & $\begin{array}{r}-.283 \\
.091\end{array}$ & .10 \\
\hline $\begin{array}{l}\text { Agotamiento } \\
\text { emocional }\end{array}$ & 23.495 & 8.413 & .490 & -.378 & & & & & - & - & - & - \\
\hline
\end{tabular}

Nota: M: Media; DE: Desviación estándar; g1: coeficiente de asimetría de Fisher; g2: coeficiente de curtosis de $\mathrm{Fisher} \mathrm{ICr}_{\mathrm{m}}{ }^{-r_{v}}$ : Intervalo de confianza para la diferencia de correlaciones; $q$ : $q$ de Cohen 


\section{Resultados}

\section{Análisis descriptivo}

Las magnitudes de asimetría $(<2)$ y curtosis $(<7)$ indican un acercamiento a la normalidad (Tabla 1). Los indicadores de confiabilidad ( $\alpha \mathrm{y} \mathrm{r}_{\mathrm{ii}}$ ) fueron aceptables para Extraversión ( $\alpha=.520 ; r_{i i}=.265$ ), Afabilidad $\left(\alpha=.669 ; r_{i i}=.403\right)$, Responsabilidad $(\alpha$ $\left.=.633 ; r_{i i}=.365\right)$, Neuroticismo $\left(\alpha=.619 ; r_{i i}=.351\right)$, Apertura $\left(\alpha=.545 ; r_{i i}=.285\right)$; y $\operatorname{AE}(\alpha=.892)$.

En cuanto a las correlaciones entre los 5GF y el agotamiento emocional en varones y mujeres, fueron obtenidas magnitudes significativas ( $r \approx$ .20) con extraversión, responsabilidad y neuroticismo en ambos grupos (ver Tabla 1), pero tanto afabilidad como apertura presentaron diferencias en cuanto a su correlación con el agotamiento emocional ( $q>$.10): en varones, afabilidad presentaba una asociación más fuerte con agotamiento emocional, mientras que apertura presentó una correlación de mayor magnitud en el grupo de las mujeres (Tabla 1), por lo que no fueron incluidos en el siguiente análisis.

\section{Análisis principal}

La evaluación de los supuestos para realizar la regresión múltiple muestra evidencia favorable: asociación lineal entre variables (tabla 1), estadístico de Durbin-Watson aceptable (1.956), aproximación razonable a la normalidad por parte de los residuos ( $Z=1.438 ; p=.032$ ), y ausencia de multicolinealidad en vista de los valores para extraversión (tolerancia $=.721 ; \mathrm{FIV}=1.387$ ), afabilidad (tolerancia $=.628 ; \mathrm{FIV}=1.592)$, responsabilidad (tolerancia $=.632 ; \mathrm{FIV}=1.583$ ), neuroticismo (tolerancia $=.971 ;$ FIV $=1.030)$, y apertura (tolerancia $=$ .717; FIV = 1.395).

En cuanto al análisis de regresión, se halló que extraversión, responsabilidad y neuroticismo explican de conjunta significativa y moderadamente $\left(R^{2}=.288\right.$; IC95\% .201, .401) la variabilidad del $\mathrm{AE}$, y solo la predicción individual $(\beta)$ de neuroticismo fue aceptable ( $\beta=.480$; IC95\% .339, .636), mientras que extraversión ( $\beta=-.063$; IC95\% -.385, .311) y responsabilidad ( $\beta=-.154$; IC95\% -.503, .137) no predicen significativamente el burnout académico dado que sus IC incluyen al cero. Sin embargo, un panorama distinto se aprecia al considerar los $r_{s^{\prime}}$ ya que los tres rasgos de personalidad predicen de forma significativa sobre el agotamiento emocional: neuroticismo ( $r s=.925$; IC95\% .814, .982), extraversión ( $r_{s}=-.550$; IC95\% -.727, -.277) y responsabilidad $\left(r_{s}=-.378\right.$; IC95\% -.535, -.168).

\section{Discusión}

El burnout es un fenómeno que puede ocasionar consecuencias en el ámbito académico, social y personal (Caballero et al., 2010), y además se ha reportado que existen factores de personalidad como el neuroticismo, que predisponen a experimentar el burnout académico. Por ello, debido a que en el contexto peruano no se han reportado estudios en esta línea de investigación, el objetivo del presente estudio es identificar cuál de los 5GF predice significativamente el burnout académico.

Las hipótesis de estudio obtuvieron soporte empírico, y si bien en un primer momento las asociaciones entre el agotamiento emocional con extraversión y responsabilidad fueron aceptables ( .20), luego del análisis multivariado realizado se identificó que su capacidad predictiva sobre el agotamiento emocional es casi nula en presencia de neuroticismo, pero al emplear un método más apropiado para el caso de predictores relacionados (e.g., $r_{s}$ ), las asociaciones propuestas inicialmente tuvieron respaldo. Es decir, si $\beta$ no es significativo y rs sí lo es, se debe a que el predictor explica parte de la varianza de la variable criterio, pero otras variables predictoras toman el crédito (Stellefson, Hanik, Chaney, \& Chaney, 2008). Esto podría explicarse debido a la asociación entre los 5GF (predictores), dado que no son independientes, y más bien comparten varianza relevante. Por ejemplo, suele existir una asociación directa entre cuatro de los $5 \mathrm{GF}$, excepto neuroticismo, con quien las demás dimensiones se asocian negativamente (para un resumen ver Dominguez-Lara, Merino-Soto, Zamudio, y Guevara-Cordero, 2018), lo que también se aprecia en estudios peruanos con el BFI-15 (Dominguez-Lara y Merino-Soto, 2018b). Por esto, un panorama distinto podría ser establecido si los predictores fueran independientes, pero la complejidad inherente al constructo personalidad evidencia lo contrario. 
Los resultados en esta investigación son convergentes con lo reportado en otros espacios (García-Izquierdo et al., 2009; Langelaan et al., 2006; Morgan, y De Bruin, 2010; Sulea et al., 2015) donde se concluye que el factor neuroticismo está asociado en mayor grado con el síndrome de burnout. Esto se puede explicar en el marco del sistema cognitivo-afectivo de la personalidad (Mischel \& Shoda, 1995), ya que altos niveles de neuroticisimo se asocian con la tendencia a seleccionar y vivenciar eventos que lo predisponen a experimentar emociones negativas (Costa \& McCrae, 1992; Emmons, Diener, y Larsen, 1985), haciendo que el entorno sea evaluado como amenazante (Langelan et al., 2006) y que no se cuenta con los recursos para afrontar tal situación y que hay una sobrecarga. Esto quiere decir que si no logra afrontar de manera funcional las adversidades, sean personales o académicas, y si cuenta con elevados niveles de neuroticismo, es más probable incrementar el agotamiento emocional, desarrollar burnout académico, y posteriormente experimentar ansiedad o depresión (Caballero et al., 2010; Escuderos et al., 2017).

Por otro lado, la asociación inversa con extraversión y responsabilidad indicarían que es relevante para el estudiante socializar con sus compañeros y contar con una red social que le brinde apoyo (extraversión), así como una vida académica disciplinada (responsabilidad) para prevenir el agotamiento emocional. Lo primero ya se ha observado en entornos laborales donde el vínculo con los colegas minimiza el impacto del burnout en el trabajo (Beltrán, Moreno, \& Reyes, 2004), lo que es posible extender al ámbito académico (Kim, Jee, Lee, An, \& Lee, 2018); y lo segundo da cuenta de que la organización de tareas y cumplimiento de las mismas en los plazos establecidos minimizaría la posibilidad de acumulación y, en consecuencia, de agotamiento emocional.

Los resultados poseen relevancia práctica al momento de planificar las evaluaciones de inicio de ciclo en las instituciones universitarias, ya que detectando a aquellos estudiantes que poseen el rasgo neuroticismo en medida más elevada, así como bajos niveles de extraversión y responsabilidad, podrían ser evaluados de forma más minu- ciosa y de ese modo prevenir la aparición del burnout académico y sus consecuencias. Desde un punto de vista teórico, se brinda información que cubre un área de vacancia en la investigación psicológica peruana, lo que posibilitará su ampliación empleando métodos de análisis más sofisticados, así como la inclusión de un mayor número de variables en el marco de modelos explicativos.

Entre las limitaciones del presente estudio podría destacar el uso de una escala breve para evaluar personalidad (Dominguez-Lara y Merino-Soto, 2018a) que, si bien pueden arrojar indicadores bajos de confiabilidad de las puntuaciones, son aceptables a decir de la amplitud del constructo evaluado y fue empleado un método analítico acorde con esta situación. Adicionalmente, dada la importancia de los tres rasgos evaluados, en futuras investigaciones podría evaluarse con la versión extensa del instrumento. Por otro lado, al no existir una evaluación de seguimiento no se puede concluir sobre el poder predictivo de los rasgos de personalidad neuroticismo, extraversión y res ponsabilidad sobre la génesis y mantenimiento del AE (e.g., Armon et al., 2012), por lo que sería conveniente implementar estudios longitudinales, dado el carácter estable de la medida de agotamiento emocional empleada en el presente estudio. A favor de la investigación cabe resaltar que los instrumentos fueron aplicados las primeras semanas de clases en el primer semestre del año, por lo que es poco probable que exista algún tipo de interferencia que podría ocurrir si la evaluación se programara para final del año. Del mismo modo, se presume que la exigencia entre carreras profesionales es similar, ya que todas pertenecen al grupo de carreras económico-empresariales, por lo que tampoco representa una limitación.

En conclusión, neuroticismo, extraversión y responsabilidad predicen de forma significativa el agotamiento emocional en estudiantes universitarios, lo que brinda mayores luces para una evaluación focalizada en esos rasgos, considerando que un afrontamiento deficiente del estrés trae consigo consecuencias nocivas en la salud psicológica (Caballero et al., 2010). 


\section{Referencias}

Alarcon, G., Eschleman, K. J., \& Bowling, N. A. (2009). Relationships between personality variables and burnout: A meta-analysis. Work \& Stress: An International Journal of Work, Health \& Organizations, 23(3), 244-263. http://dx.doi. org/10.1080/02678370903282600

Arias-Gundín, O., \& Vizoso, C. M. (2018). Relación entre estrategias activas de afrontamiento, burnout y engagement en futuros educadores. Estudios sobre Educación, 35, 409-427. http://dx.doi. org/10.15581/004.34.409-427

Armon, G.., Shirom, A., \& Melamed, S. (2012). The Big Five personality factors as predictors of changes across time in burnout and its facets. Journal of Personality, 80(2), 403-427, http://dx.doi.org/10.1111/j.14676494.2011.00731.x

Asociación Médica Mundial (1964). Declaración de Helsinki. Helsinki: AMM. http://www.conamed.gob.mx/prof_ salud/pdf/helsinki.pdf

Ato, M., López, J. y Benavente, A. (2013). Un sistema de clasificación de los diseños de investigación en psicología. Anales de Psicología, 29(3), 1038-1059. http://dx.doi:10.6018/analesps.29.3.178511

Beltrán, C. A., Moreno, M. P., \& Reyes, M. B. P. (2004). Apoyo social y síndrome de quemarse en el trabajo o burnout: una revisión. Psicología y salud, 14(1), 79-87. http://psicologiaysalud.uv.mx/index.php/ psicysalud/article/view/868/1595

Benet-Martínez, V., \& John, O. P. (1998). Los Cinco Grandes across cultures and ethnic groups: Multitraitmultimethod analyses of the Big Five in Spanish and English. Journal of Personality and Social Psychology, 75(3), 729-750 http://dx.doi. org/10.1037/0022-3514.75.3.729

Boysan, M., \& Kiral, E. (2016). Associations between procrastination, personality, perfectionism, selfesteem and locus of control. British Journal of Guidance \& Counselling, 45(3), 284-296. http://dx.doi. org/10.1080/03069885.2016.1213374

Caballero, C. C., Hederich, C., \& Palacio, J. E. (2010). El burnout académico: delimitación del síndrome y factores asociados con su aparición. Revista Latinoamericana de Psicología, 42(1), 131-146. http://www.scielo.org. co/pdf/rlps/v42n1/v42n1a12.pdf

Caprara, G.V., Vecchione, M., Alessandri, G., Gerbino, M., \& Barbaranelli, C. (2011). The contribution of personality traits and self-efficacy beliefs to academic achievement: A longitudinal study. British Journal of Educational Psychology, 81, 78-96. http:// dx.doi.org/10.1348/2044-8279.002004

Cebrià, J., Segura, J., Corbella, S., Sos, P., Comas, O., García, M., ... Pérez, J. (2001). Rasgos de personalidad y burnout en médicos de familia. Atención Primaria, 27(7), 459468. http://dx.doi.org/10.1016/S0212-6567(01)78836-1

Clark, M. H., \& Schroth, C. A. (2010). Examining relationships between academic motivation and personality among college students. Learning and individual differences, 20(1), 19-24. http://dx.doi.org/10.1016/j. lindif.2009.10.002

Clark, L. A., \& Watson, D. (1995). Constructing validity: Basic issues in objective scale development. Psychological Assessment, 7(3), 309 - 319. http://www.bwgriffin. com/gsu/courses/edur9131/content/Clark_validity_ scaledevelopment.pdf

Cohen, J. (1992). A power primer. Psychological Bulletin, 112(1), 155 - 159. http://dx.doi.org/10.1037/00332909.112.1.155

Colegio de Psicólogos del Perú (2017). Código de ética y deontología. https://www.cpsp.pe/documentos/ marco_legal/codigo_de_etica_y_deontologia.pdf

Costa, P. T., \& McCrae, R. R. (1992). Normal personality assessment in clinical practice: The NEO personality inventory. Psychological Assessment, 4, 5-13. http:// dx.doi.org/10.1037/1040-3590.4.1.5

Courville, T., \& Thompson, B. (2001). Use of structure coefficients in published multiple regression articles: $\beta$ is not enough. Educational and Psychological Measurement, 61(2), 229-248. http:// dx.doi.org/10.1177/0013164401612006

Da Silva, R., Tonini, C., Dias, L., Serrano, P., Siqueira, C., \& Guido, L. (2014). Hardy personality and burnout syndrome among nursing students in three Brazilian universities - an analytic study. BMC Nursing, 19(9), 1-6. http://dx.doi.org/10.1186/1472-6955-13-9

De Feyter, T., Caers, R., Vigna, C., \& Berings, D. (2012). Unraveling the impact of the Big Five personality traits on academic performance: The moderating and mediating effects of self-efficacy and academic motivation. Learning and Individual Differences, 22, 439-448. http://dx.doi.org/10.1016/j. lindif.2012.03.013

Dominguez-Lara, S. (2018). Agotamiento emocional académico en estudiantes universitarios: ¿cuánto influyen las estrategias cognitivas de regulación emocional? Educación Médica, 19(2), 96 - 103. https:// 


\section{doi.org/10.1016/j.edumed.2016.11.010}

Dominguez-Lara, S., \& Merino-Soto, C. (2018a). Dos versiones breves del Big Five Inventory en universitarios peruanos: BFI-15p y BFI-10p. Liberabit, 24(1), 81 - 96. http://dx.doi.org/10.24265/liberabit.2018.v24n1.06

Dominguez-Lara, S., \& Merino-Soto, C. (2018b). Estructura interna del BFI-10P y BFI-15P: un estudio complementario con enfoque CFA y ESEM. Revista Argentina de Ciencias del Comportamiento, 10(3), 22 - 34. https://revistas.unc.edu.ar/index.php/racc/ article/view/21037

Dominguez-Lara, S., Moscoso, M., Merino-Soto, C., \& NavarroLoli, J. (2016). Un método para la comparación de correlaciones basado en intervalos de confianza: aportes a Tejedor et al. Revista de Psiquiatría y Salud Mental, 9(4), 228 - 229. http://dx.doi.org/10.1016/j. rpsm.2015.12.001

Dominguez-Lara, S., Merino-Soto, C., Zamudio, B., \& Guevara-Cordero, C. (2018). Big Five Inventory en Universitarios Peruanos: Resultados Preliminares de su Validación. Psykhe, 27(2), 1 -12. http://dx.doi. org/10.7764/psykhe.27.2.1052

Dominguez-Lara, S., Fernández-Arata, M., ManriqueMillones, D., Alarcón-Parco, D., \& Díaz-Peñaloza, M. (2018). Datos normativos de una escala de agotamiento emocional académico en estudiantes universitarios de psicología de Lima (Perú). Educación Médica, 19(3), 246 - 255. http://dx.doi. org/10.1016/j.edumed.2017.09.002

Duru, E., Duru, S., \& Balkis, M. (2014). Analysis of relationships among burnout, academic achievement, and self-regulation. Educational Sciences: Theory \& Practice, 14(4), 1274-1284. https://dx.doi.org/10.12738 / estp.2014.4.2050

Ellis, P. (2010). The essential guide to effect sizes: Statistical power, meta-analysis, and the interpretation of research results. Cambridge University Press.

Emmons, R. A., Diener, E. D., \& Larsen, R. J. (1985). Choice of situations and congruence models of interactionism. Personality and Individual Differences, 6(6), 693-702. https://doi.org/10.1016/0191-8869(85)90080-7

Escuderos, A. M., Colorado, Y. S., \& Sañudo, J. P. (2017). Burnout académico y síntomas relacionados con problemas de salud mental en universitarios colombianos. Psychologia. Avances de la disciplina, 11(2), 51-72. http://www.scielo.org.co/pdf/ psych/v11n2/1900-2386-psych-11-02-00051.pdf

Ferrel, F. R., Ferrel, L. F., Cantillo, A. A., Jaramillo, J., \& Jiménez, S. M. (2017). Variables académicas y sociodemográficas relacionadas con el Síndrome de Burnout, en estudiantes de Ingenierías y Ciencias de la Salud de una universidad estatal de Colombia. Psicogente, 20(38), 336-352. http://dx.doi. org/10.17081/psico.20.38.2555

Ferguson, C.J. (2009). An effect size primer: a guide for clinicians and researchers. Professional Psychology: Research and Practice, 40(5), 532-538. http://dx.doi. org/10.1037/a0015808

Finney, S. J. \& DiStefano C. (2006). Non-normal and categorical data in structural equation modeling. In G. R. Hancock \& R. O. Mueller (Eds.), Structura Equation Modeling. A Second Course (pp. 269-314). Greenwich, CT: Information Age Publishing.

Fontana, S. A. (2011). Estudio preliminar de las propiedades psicométricas de la escala de Desgaste Emocional para estudiantes universitarios. Revista Argentina de Ciencias del Comportamiento, 3(2), 44-48. https://revistas.unc.edu.ar/index.php/racc/article/ view/5227

Galbraith, C. S., \& Merrill, G. B. (2012). Academic and WorkRelated Burnout: A Longitudinal Study of Working Undergraduate University Business Students. Journal of College Student Development, 53(3), 453463. http://dx.doi.org/10.1353/csd.2012.0044

Galbraith, C. S., \& Merril, G. B. (2015). Academic performance and burnout: an efficient frontier analysis of resource use efficiency among employed university students. Journal for Further and Higher Education, 39(2), 255277. http://dx.doi.org/10.1080/0309877X.2013.858673

García-Izquierdo, A. L., Ramos-Villagrasa, P. J., \& GarcíaIzquierdo, M. (2009). Los Big Five y el Efecto Moderador de la Resistencia en el Agotamiento Emocional. Revista de Psicología del Trabajo y de las Organizaciones, 25(2), 135-147. http://scielo.isciii.es/ pdf/rpto/v25n2/v25n2a04.pdf

Garrosa, E., Rainho, C., Moreno-Jiménez, B., \& Monteiro, M. J. (2010). The relationship between job stressors , hardy personality, coping resources and burnout in a sample of nurses: A correlational study at two time points. International Journal of Nursing Studies, 47, 205-215. http://dx.doi.org/10.1016/j. ijnurstu.2009.05.014

González, R., Souto, A., Fernández, R., \& Freire, C. (2011). Regulación emocional y burnout académico en estudiantes universitarios de Fisioterapia. Revista de Investigación en Educación, 9(2), 7-18. http://reined. webs.uvigo.es/index.php/reined/article/view/111

Hortigüela, D. \& Pérez-Pueyo, A. (2016). Influencia de la 
regulación del trabajo del alumnado universitario en la implicación hacia las tareas. Psychology, Society, \& Education, 8(1), 39-51. http://dx.doi.org/10.25115/ psye.v8i1.458

Jacobs, S. R., \& Dodd, D. K. (2003). Student Burnout as a Function of Personality, Social Support, and Workload. Journal of College Student Development, 44(3), 291-303. http://dx.doi.org/10.1353/ csd.2003.0028

Jackson, E. R., Shanafelt, T. D., Hasan, O., Satele, D. V., \& Dyrbye, L. N. (2016). Burnout and alcohol abuse/ dependence among U.S. medical students. Academic Medicine, 91(9), 1251-1256. http://dx.doi.org/10.1097/ ACM.0000000000001138

Kim, B., Jee, S., Lee, J., An, S., \& Lee, S. M. (2018). Relationships between social support and student burnout: A meta analytic approach. Stress and Health, 34(1), 127-134. http://dx.doi.org/10.1002/smi.2771

Kristensen, T. S., Borritz, M., Villadsen, E., \& Christensen, K. B. (2005). The Copenhagen Burnout Inventory: A new tool for the assessment of burnout. Work \& Stress, 19(3), 192- 207. http://dx.doi. org/10.1080/02678370500297720

Lang, F. R., John, D., Lüdtke, O., Schupp, J., \& Wagner, G. G. (2011). Short assessment of the Big Five: Robust across survey methods except telephone interviewing. Behavior Research Methods, 43(2), 548-567. http://dx.doi.org/10.3758/s13428-011-0066-z

Langelaan, S., Bakker, A. B., van Doornen, L. J., \& Schaufeli, W. B. (2006). Burnout and work engagement: Do individual differences make a difference? Personality and Inidividual Differences, 40, 521-532. http://dx.doi.org/10.1016/j.paid.2005.07.009

Lee, S. J., Choi, Y. J., \& Chaeb, H. (2017). The effects of personality traits on academic burnout in Korean medical students. Integrative Medicine Research, 6(2), 207-213. http://dx.doi.org/10.1016/j. imr.2017.03.005

Lorenzo-Seva, U., Ferrando, P.J., \& Chico, E. (2010). Two SPSS programs for interpreting multiple regression results. Behavioral Research Methods, 41(1), 29-35. http://dx.doi.org/10.3758/BRM.42.1.29

Martínez, I. M., \& Marques, A. (2005). Burnout en estudiantes universitarios de España y Portugal y su relación con variables académicas. Altheia, 21, 21-30. http:// pepsic.bvsalud.org/pdf/aletheia/n21/n21a03.pdf

Maslach, C. (2003). Job burnout: new directions in research and intervention. Current Directions in Psychological Science, 12(5), 189-192. https://doi.

\section{org/10.1111/1467-8721.01258}

Maslach, C., \& Leiter, M. P. (2016). Understanding the burnout experience: recent research and its implications for psychiatry. World Psychiatry, 15(2), 103-111. http:// dx.doi.org/10.1002/wps.20311

McGeown, S.P., Putwain, D., Simpson, E.G., Boffey, E., Markham, J., \& Vince, A. (2014). Predictors of adolescents' academic motivation: Personality, selfefficacy and adolescents' characteristics. Learning and Individual Differences, 32, 278-286. http:// dx.doi.org/10.1016/j.lindif.2014.03.022

McIIloy, D., Poole, K., Ursavas O.M., \& Moriarty, A. (2015). Distal and proximal associates of academic performance at secondary level: A mediation model of personality and self-efficacy. Learning and Individual Differences, 38, 1-9. http://dx.doi.org/10.1016/j. lindif.2015.01.004

Mischel, W., \& Ayduk, O. (2002). Self-regulation in a cognitive-affective personality system: Attentional control in the service of the self. Self and Identity. Special Issue: Self and Identity, 1, 113-120. http:// dx.doi.org/10.1080/152988602317319285

Mischel, W., \& Shoda, Y. (1995). A cognitive-affective system theory of personality: Reconceptualizing situations, dispositions, dynamics, and invariance in personality structure. Psychological Review, 102, 246-268. http://dx.doi.org/10.1037/0033 $295 \times .102 .2 .246$

Moreno, B., Morett, N., Rodríguez, A., \& Morante, M. (2006). La personalidad resistente como variable moduladora del síndrome de burnout en una muestra de bomberos. Psicothema, 18(3), 413-418. www. psicothema.es/pdf/3231.pdf

Morgan, B., \& De Bruin, K. (2010). The relationship between the big five personality traits and burnout in South African university students. South African Journal of Psychology, 40(2), 182-191. http://dx.doi. org/10.1177/008124631004000208

Nikodijević, A., Labrović, J. A., \& Đoković, A. (2012). Academic Burnout Among Students at Faculty of Organizational Sciences. Education Management, 565 - 570. https://dx.doi.org/10.7595/management. fon.2012.0019

Ortega, M. E., Ortiz, G., \& Coronel, P. (2006). Burnout en médicos y enfermeras y su relación con el constructo de personalidad resistente. Psicología y Salud, 17(1), 5-16. https://revistas.uv.mx/index.php/psicysalud/ article/view/730

Pagnin, D., de Queiroz, V., Santos, Y. T., Soares, A. S., Bastos, M., 
\& Thomasin, T. (2014). The relation between burnout and sleep disorders in medical students. Academic Psychiatry, 38(4), 438-444. http://dx.doi.org/10.1007/ s40596-014-0093-z

Palacios, J. E., Caballero, C. C., González, O., Gravini, M., \& Contreras, K. P. (2012). Relación del burnout y las estrategias de afrontamiento con el promedio académico en estudiantes universitarios. Universitas Psychologica, 11(2), 535-544. https:// revistas.javeriana.edu.co/index.php/revPsycho/ article/view/583

Pereira-Lima, K., \& Loureiro, S. R. (2015). Burnout, anxiety, depression and social skills in medical residents. Psychology, Health \& Medicine, 20(3):353 - 362. http://dx.doi.org/10.1080/13548506.2014.936889

Polo, J. D., Santiago, V., Navarro, M. C., \& Alí, A. (2016). Creencias irracionales, síndrome de Burnout y adicción al trabajo en las organizaciones. Psicogente, 19(35), 148-160. http://dx.doi.org/10.17081/psico.19.35.1215

Rammstead, B., \& Beierlein, C. (2014). Can't we make ir any shorter? Journal of Individual Differences, 35(4), 212 - 220. http://dx.doi.org/10.1027/1614-0001/a000141

Ramos, F., Manga, D., \& Morán, C. (2005). Escala de cansancio emocional (ECE) para estudiantes universitarios: Propiedades psicométricas y asociación. Interpsiquis. http://www.psiquiatria.com/articulos/ estres/20478/.

Salanova, M., Martínez, I., Bresó, E., Llorens, S., \& Grau, R. (2005). Bienestar psicológico en estudiantes universitarios: facilitadores y obstaculizadores del desempeño académico. Anales de Psicología, 21, 170 180. https://www.um.es/analesps/v21/v21_1/16-21_1. pdf

Salanova, M., Bresó, E., \& Schaufeli, W. B. (2005). Hacia un modelo espiral de las creencias de eficacia en el estudio del burnout y del engagement. Ansiedad y Estrés, 11(2-3), 215-231.

Salanova, M., Schaufeli, W., Martínez, I., \& Bresó, E. (2010). How obstacle and facilitators predict academic performance: the mediation role of study burnout and engagement. Anxiety, Stress, and Coping, 23(1), 53-70. https://doi.org/10.1080/10615800802609965
Schaufeli, W. B., Martínez, I. M., Marques, A., Salanova, M., \& Bakker, A. B. (2002). Burnout and Engagement in University Students: A Cross-National Study. Journal of Cross-Cultural Psychology, 33(5), 464-481. http:// dx.doi.org/10.1177/0022022102033005003

Skodova, Z., Lajciakova, P., \& Banovcinova, L. (2017). Burnout syndrome among health care students: the role of type personality. Western Journal of Nursing Research, 39(3), 416-429. http://dx.doi org/10.1177/0193945916658884

Stellefson, M.L., Hanik, B.W., Chaney, B.H., \& Chaney, J.D. (2008). A tutorial on calculating and interpreting regression coefficients in health behavior research. The Health Educator, 40(1), 12-20. https://files.eric. ed.gov/fulltext/EJ863505.pdf

Sulea, C., van Beek, I., Sarbescu, P., Virga, D., \& Schaufeli, W. (2015). Engagement, boredom, and burnout among students: basic need satisfaction matters more than personality traits. Learning and Individual Differences, 42, 132-138. http://dx.doi.org/10.1016/j. lindif.2015.08.018

Tuithof, M., ten Have, M., Beekman, A., van Dorsselaer, S., Kleinjan, M., Schaufeli, W., \& de Graaf, R. (2017). The interplay between emotional exhaustion, common mental disorders, functioning and health care use in the working population. Journal of Psychosomatic Research, 100, 8-14. http://dx.doi.org/10.1016/j. jpsychores.2017.06.018

Vilà Baños, R., Torrado-Fonseca, M., y Reguant-Álvarez, M. (2019). Análisis de regresión lineal múltiple con SPSS: un ejemplo práctico. REIRE Revista d'Innovació i Recerca en Educació, 12(2), 1-10. http:// doi.org/10.1344/reire2019.12.222704

Yu, J. H., Chae, S. J., \& Chang, K. H. (2016). The relationship among self-efficacy, perfectionism and academic burnout in medical school students. Korean Journal of Medical Education, 28(1), 49-55. http://dx.doi. org/10.3946/kjme.2016.9

Zou, G. (2007). Toward using confidence intervals to compare correlations. Psychological Methods, 12(4), 39-413. http://dx.doi.org/10.1037/1082-989X.12.4.399

RIDU / Revista Digital de Investigación en Docencia Universitaria / e-ISSN: 2223-2516

(C) Los autores. Este artículo es publicado por la Revista Digital de Investigación en Docencia Universitaria del Área de Institutional Research and Effectiveness de la Dirección de Aseguramiento de la Calidad, Universidad Peruana de Ciencias Aplicadas. Este es un artículo de acceso abierto, distribuido bajo los términos de la LicenciaCreativeCommons Atribución-CompartirIgual 4.0 Internacional.(http://creativecommons.org/licenses/by-sa/4.0/), que permite el uso no comercial, distribución y reproducción en cualquier medio, siempre que la obra original sea debidamente citada. 